\title{
A Dosimetric Comparison of Primary Chemoradiation Versus Postoperative Radiation for Locally Advanced Oropharyngeal Cancer
}

\author{
Stanley K. Woo ${ }^{1}$, Chad Freeman ${ }^{1}$, Brock J. Debenham ${ }^{2}$ \\ 1. Department of Oncology, University of Alberta 2. Department of Oncology, University of Alberta, \\ Edmonton, CAN
}

$\square$ Corresponding author: Brock J. Debenham, debenham@ualberta.ca

Disclosures can be found in Additional Information at the end of the article

\section{Abstract}

\section{Introduction}

Advanced-stage oropharyngeal cancer can be treated with primary chemoradiation (CRT) or primary surgery with adjuvant radiotherapy, both with similar survival outcomes. Though primary CRT prescribes a higher dose, adjuvant radiation requires irradiating the surgical bed, which may increase the high dose planned target volume (PTV). We hypothesize that the integral dose to the neck and dose to critical structures will be lower with primary CRT than adjuvant radiotherapy.

\section{Methods}

We selected the last 18 patients who underwent surgery and adjuvant radiotherapy at one institution between July 2015 and August 2016 with American Joint Committee on Cancer (AJCC) stage III or IVA oropharyngeal squamous cell cancer. Primary CRT treatment plans were created on the patients' preoperative computed tomography (CT) scans and prescribed 70 Gy in 33 fractions, while postoperative plans were prescribed $60 \mathrm{~Gy}$ in 30 fractions. The radiation doses received by organs at risk for each primary CRT plan were compared to the corresponding adjuvant radiation plan.

\section{Results}

Primary CRT plans had significantly smaller high dose PTV than adjuvant radiation plans (187.3 cc (95\% CI 134.9-239.7) and 466.3 cc (95\% CI 356.7-575.9), p<0.0001). The neck integral dose was lower in 14 of 18 plans using primary CRT, although this was not statistically significant ( $\mathrm{p}=0.5375$ ). The primary CRT plans had lower mean doses to ipsilateral (31.8 Gy (95\% CI 27.5-36.0) vs $39.3 \mathrm{~Gy}$ (95\% CI 35.4-43.1), $\mathrm{p}=0.0009)$ ) and contralateral parotid glands (22.5 Gy (95\% CI 22.1-22.8) vs 27.6 Gy (95\% CI 23.4-31.8), p=0.0238) and larynx (20.7 Gy (95\% CI 19.3-22.2) vs 40.2 Gy (95\% CI 30.8-46.6), p<0.0001).

\section{Conclusion}

Primary CRT offered a decreased neck integral dose, though it was statistically insignificant. Primary CRT plans reduce mean dose to larynx and parotid glands in comparison to postoperative radiation, which may result in lower toxicities. Clinical trials comparing primary CRT and primary surgery are warranted to compare patient toxicities. 
Categories: Otolaryngology, Radiation Oncology, Oncology

Keywords: oropharyngeal cancer, radiation, surgery, head and neck cancer, head and neck cancer, hpv

\section{Introduction}

Approximately $70 \%$ of oropharyngeal cancer patients present at advanced stages [1]. For these patients, two common treatment modalities exist: primary surgery with postoperative radiation (with or without chemotherapy) and primary chemoradiation (with or without salvage surgery). Both modalities achieve comparable survival outcomes for advanced stage oropharyngeal patients [2], but there is still no consensus on a preferred treatment modality.

Modalities are selected based on anatomic location, patient factors/values, and physician influences. Despite similar tumour control rates, surgery causes significantly more complications that require remedial surgery, such as fistulas or permanent gastrostomies [3]. Boscolo-Rizzo et al. [4] found that chemoradiation had significantly higher long-term quality of life scores than surgery with postoperative radiation. A retrospective single centre study found that surgery followed by chemoradiation gave the patient population the best survival rates compared to surgery with postoperative radiotherapy or chemoradiotherapy alone [5]; however, combining more treatment modalities often increases patient morbidity. Therefore, a trade off exists between tumour control and reduced side effects that physicians and patients must consider before selecting a treatment.

Before selecting a preferred modality, patients must consider the radiation dose to organs at risk (OAR) in the head and neck, such as the parotid glands, larynx, and mandible. While primary chemoradiation (CRT) prescribes a higher dose, adjuvant radiation (RT) may deliver more radiation to OAR because the entire post-surgical bed requires irradiation. Irradiating larger tissue volumes can increase the number and severity of side effects.

We hypothesize that the integral dose to the neck and dose to critical structures will be lower with primary CRT than adjuvant radiotherapy. Evaluating the difference in mean dose and maximum dose to OAR and the total integral dose between CRT and adjuvant RT will provide additional insight into the optimal treatment modality for locally advanced oropharyngeal cancer patients.

\section{Materials And Methods}

The study was submitted for a Research Ethics Board (REB) review and follows the Tri-Council Policy Statement: Ethical Conduct for Research Involving Human Subjects (TCPS2), as data collection involved retrospective patient information such as computed tomography (CT) scans and radiotherapy treatment plans from the Cross Cancer Institute (Edmonton, Canada). The REB determined that ethics approval was not required as this is a quality improvement based study.

Using a retrospective cohort, we compared the total integral dose and dose to OAR that patients received when they were treated with adjuvant RT to a theoretical CRT plan using their preoperative CT scans. This study included both an experimental and control group as summarized in Figure 1. 


\section{Cureus}

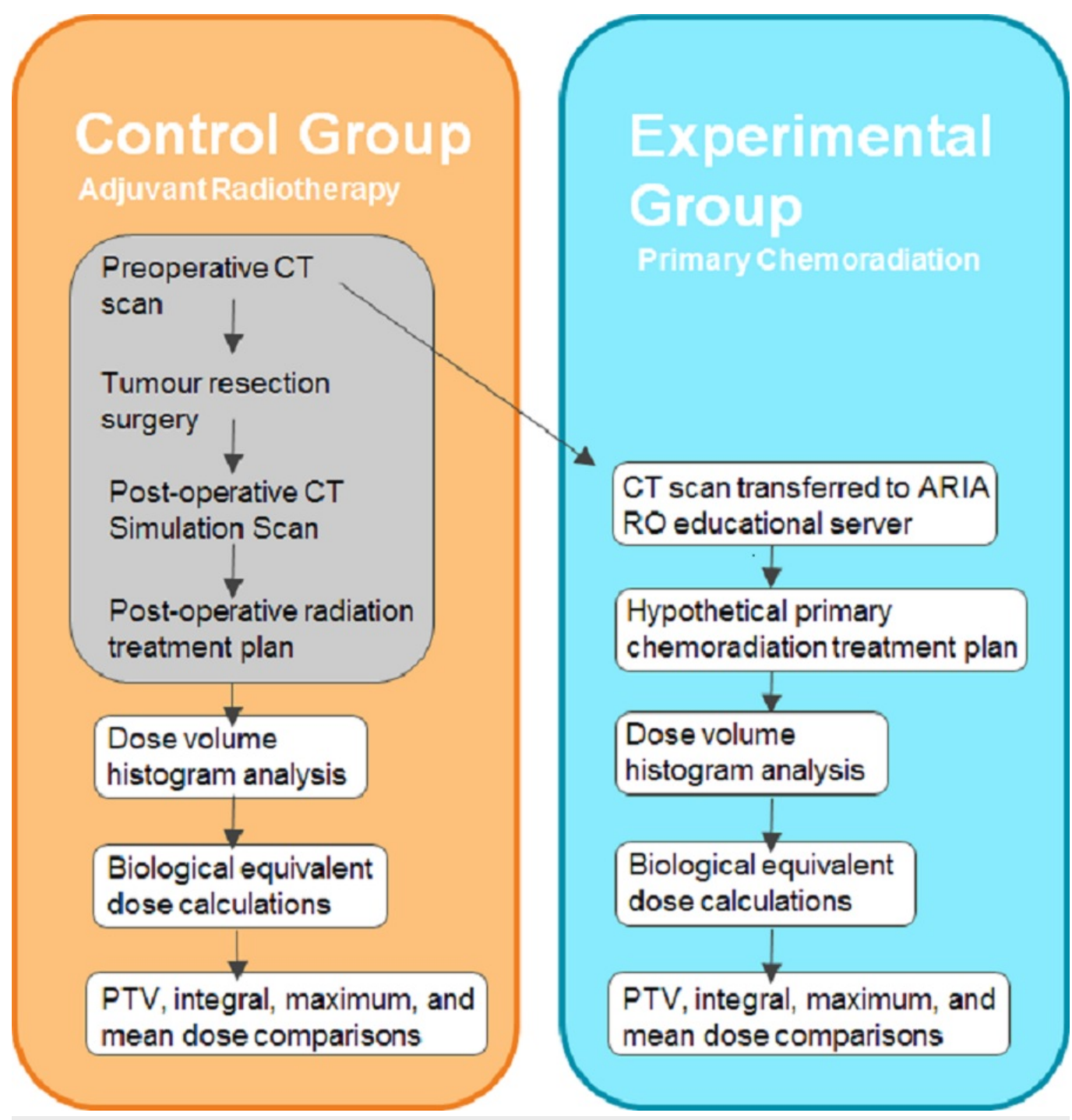

\section{FIGURE 1: Experimental Design}

Description of our methodology and how we defined our control and experimental group. The control were the actual adjuvant RT plans, whereas the experimental group were the primary CRT plans. The grey shaded rectangle represents steps that were completed prior to this study.

( $\mathrm{CT}=$ computed tomography, $\mathrm{PTV}=$ planning tumor volume, $\mathrm{RO}=$ radiation oncology $)$.

The last 20 consecutive patients with American Joint Committee on Cancer (AJCC) stage III or IVA oropharyngeal cancer who underwent primary surgery and postoperative radiotherapy for their disease at our centre between July 2015 and August 2016 were included in our study. Apart from the tumour resection surgery, patients had no previous head and neck surgeries for malignancies and no previous head and neck radiotherapy treatments.

We obtained the postoperative radiotherapy plans (60 Gy in 30 fractions delivered daily) and dose-volume-histograms (DVH) from the patients noted above to serve as our control group. Our standard procedure is to treat the post-surgical bed and involved lymph node levels to 60 $\mathrm{Gy}$, and the uninvolved neck to $54 \mathrm{~Gy}$. The radiation treatments were planned and completed at the Cross Cancer Institute. Each patient's plans and images were anonymized and assigned a random identification number. 
For our experimental group, the patients' preoperative diagnostic CT scans were transferred to the ARIA ${ }^{\circledR}$ oncology information system for radiation oncology, which contains the radiotherapy treatment planning software used clinically at the Cross Cancer Institute. The parotid glands, submandibular glands, mandible, esophagus, larynx, and pharyngeal constrictor muscles were contoured as critical structures using the contouring guidelines from the Radiation Therapy Oncology Group (RTOG) protocol 1016. The RTOG 1016 protocol contains planning guidelines and objectives for target volumes and critical structures for primary chemoradiation of advanced stage oropharyngeal patients. A treatment couch structure was added to each plan to simulate the radiotherapy units. We generated volumetric arc therapy plans, which was the same technique used in postoperative plans, with the Eclipse 3D planning system (version 13.6, Varian Medical Systems, Palo Alto, CA). The dose prescription was different, 70 Gy in 33 fractions delivered daily, but the dose algorithm and plan normalization ( $95 \%$ of PTV received $100 \%$ of the dose) were maintained with the adjuvant RT plans to provide intrapatient consistency. Normal tissue optimizations were the same between postoperative and preoperative planning to reduce bias. Clinical target volume (CTV) to PTV margins were $0.5 \mathrm{~cm}$ in both groups. To account for the differences in fractionation, integral doses and OAR doses were converted to equivalent doses in 2 Gy fractions (EQD2).

As a form of quality assurance, a head and neck radiation oncologist and dosimetrist from the Cross Cancer Institute reviewed each contour and plan. The constructed primary CRT plan was compared to the patient's postoperative plan to assess the difference in integral dose and dose to OARs. Integral dose to the overall neck was calculated by multiplying the volume between the cochleas and the cricoid cartilage with the mean dose of that volume.

Data were analyzed using paired T-tests to determine any significant differences between the two regimes for each patient. A p-value of $<0.05$ was taken to be statistically significant. To determine our sample size, it was assumed that there would be a difference in integral dose between the two groups of $15 \%$ with a standard deviation of 10 for each group. Assuming a type I error of 0.05 and a type II error of 0.20 , we required a total of 20 patients for statistical significance.

\section{Results}

Data were collected for 20 patients, but two patients were omitted from analysis because of positional and scan size issues. Therefore, 18 patients were analyzed. Table 1 lists the patient characteristics of our sample group. 


\section{Cureus}

\begin{tabular}{ll} 
Demographic & Characteristics \\
\hline Age & Median 64 (range $46-76)$ \\
Sex & $94.7 \%$ male \\
Subsite & $50 \%$ base of tongue $27.8 \%$ tonsil $22.2 \%$ base of tongue and tonsil \\
Clinical AJCC stage & $5.3 \%$ III $94.7 \%$ IVA \\
p16 status & $83.3 \%$ positive $16.7 \%$ negative \\
Extracapsular extension (ECE) & $66.7 \%$ positive $33.3 \%$ negative \\
Lymphovascular invasion (LVI) & $38.9 \%$ positive $61.1 \%$ negative \\
Perineural invasion (PNI) & $33.3 \%$ positive $66.7 \%$ negative \\
Positive margins & $22.2 \%$ positive $77.8 \%$ negative \\
Percutaneous endoscopic gastrostomy tube inserted & $44.4 \%$ after surgery (before RT) $11.1 \%$ during RT
\end{tabular}

TABLE 1: Patient Demographics, Pathology, and Surgery Results

The average high dose PTV volumes for the plans made on the patients' preoperative CT scans were $40.2 \%$ smaller compared to the postoperative plans, as seen in Table 2.

\begin{tabular}{|c|c|c|c|}
\hline & Primary RT & Adjuvant RT & $P$ value \\
\hline High dose PTV volume (cc) & 187.3 (95\% Cl 134.9-239.7) & $466.3(95 \%$ Cl 356.7-575.9) & $p<0.0001$ \\
\hline Integral dose to the neck (Gy*L) & $152.6(95 \%$ Cl 130.3-174.9) & 156.6 (95\% Cl 134.7-178.5) & $p=0.5375$ \\
\hline
\end{tabular}

\section{TABLE 2: Comparison of High Dose PTV Volumes and Integral Dose to the Head and Neck Region Between the Primary RT Cohort and Adjuvant RT Cohort}

Note: All doses converted to 2 Gy fraction equivalent. P-value $</=0.05$ indicates statistical significance.

The DVH comparison of the control group versus experimental plans revealed significant differences. Table 3 contains the mean and maximum dose averages for the critical structures analyzed between the two treatment groups. The maximum doses for the ipsilateral parotid gland, the mandible, the pharyngeal constrictor muscles, and the spinal cord were significantly lower for the adjuvant RT group. Also, the mean dose to the spinal cord was also significantly lower for the adjuvant RT group. On average, the primary CRT group had lower mean doses for the ipsilateral and contralateral parotid glands, esophagus, larynx, and mandible, but only doses to the ipsilateral and contralateral parotid glands and larynx were significantly lower than the adjuvant RT plans. The primary CRT group also had lower maximum doses for the contralateral parotid and oesophagus, but these differences were not statistically significant. 


\section{Cureus}

The difference in the mean pharyngeal constrictor dose and max larynx dose was only 0.4 cGy and $1.1 \mathrm{cGy}$, respectively. Submandibular glands were resected for most patients so their dose comparisons were omitted. For individual patient doses, refer the Appendix.

\begin{tabular}{|c|c|c|c|}
\hline Structure & Primary CRT (Gy) & Adjuvant RT (Gy) & $P$ value \\
\hline Maximum dose to ipsilateral parotid & 76.8 (95\% Cl 74.8-78.7) & $65.7(95 \% \mathrm{Cl} 64.4$ to 67.0$)$ & $p<0.0001$ \\
\hline Mean dose to ipsilateral parotid & $31.8(95 \%$ Cl 27.5-36.0) & $39.3(95 \%$ Cl 35.4-43.1) & $p=0.0009$ \\
\hline Maximum dose to contralateral parotid & 56.2 (95\% Cl 52.3-59.8) & $58.4(95 \%$ Cl 52.6-64.1) & $p=0.4566$ \\
\hline Mean dose to contralateral parotid & $22.5(95 \%$ Cl 22.1-22.8) & $27.6(95 \% \mathrm{Cl} 23.4-31.8)$ & $p=0.0238$ \\
\hline Maximum dose to esophagus & 46.4 (95\% Cl 42.3-50.6) & 50.8 (95\% Cl 46.4-55.2) & $p=0.1266$ \\
\hline Mean dose to esophagus & $24.1(95 \%$ Cl $21.9-26.3)$ & $29.7(95 \%$ Cl 23.3-36.1) & $p=0.0547$ \\
\hline Maximum dose to larynx & 59.5 (95\% Cl 53.2-65.8) & 60.6 (95\% Cl 58.8-62.5) & $p=0.7307$ \\
\hline Mean dose to larynx & 20.7 (95\% Cl 19.3-22.2) & 40.2 (95\% Cl 30.8-46.6) & $p<0.0001$ \\
\hline Maximum dose to mandible & 75.7 (95\% Cl 72.9-78.6) & 65.3 (95\% Cl 64.9-65.7) & $p<0.0001$ \\
\hline Mean dose to mandible & $37.8(95 \%$ Cl 35.3-40.3) & $40.6(95 \% \mathrm{Cl} 38.0-43.2)$ & $p=0.1010$ \\
\hline Maximum dose to pharyngeal constrictors & 77.4 (95\% Cl 76.4-78.3) & 64.7 (95\% Cl 64.0 - 65.4) & $p<0.0001$ \\
\hline Mean dose to pharyngeal constrictors & 56.4 (95\% Cl 52.9-59.8) & 56.8 (95\% Cl 55.0-58.7) & $p=0.7745$ \\
\hline Maximum dose to spinal cord & $43.0(95 \% \mathrm{Cl} 42.3-43.7)$ & $40.9(95 \% \mathrm{Cl} 40.1-41.6)$ & $p<0.0001$ \\
\hline Mean dose to spinal cord & $31.6(95 \%$ Cl 30.4-32.9) & $21.1(95 \% \mathrm{Cl} 19.2-22.9)$ & $p<0.0001$ \\
\hline
\end{tabular}

\section{TABLE 3: Comparison of Mean and Maximum Doses to Critical Structures Between the Primary RT and Adjuvant RT Cohort}

Note: All doses converted to 2 Gy per fraction equivalent. P-value $</=0.05$ indicates statistical significance.

\section{Discussion}

Radiation therapy is commonly used to treat advanced stage oropharyngeal cancer, whether it be used adjuvant to surgery or as the primary modality along with chemotherapy [2]. Beyond survival rates, there are very few studies that compare these two regimes. There is lack of randomized trials investigating quality of life following treatment with chemoradiation or surgery with postoperative radiation; therefore, a preferential treatment option still does not exist for these patients [2-4]. Though Tillman et al. [6] studied a different tumour site with a different method, their results are consistent with ours in that their postoperative RT cohort had a larger PTV, and OARs such as the heart and lungs received a higher dose. Our study provides a similar dosimetric comparison and analysis that suggests that additional controlled studies are needed to further inform the patient's decision between these two treatment methods. As predicted, our study showed a significantly smaller mean high dose PTV for the primary CRT cohort, which was hypothesized to result in a lower integral dose. The primary 
CRT plans on average had lower integral doses. In fact, 14 out of 18 primary CRT plans had lower integral doses than their corresponding postoperative plans, although this was not significantly different, possibly due to the small number of patients in this study. Conversely, the results suggest that adjuvant RT is not advantageous over primary CRT in regards to delivering lower integral doses to a patient's normal tissues.

The mean ipsilateral and contralateral parotid gland dose was reduced by $19 \%$ and $18.5 \%$ in the primary CRT cohort, respectively. This reduction in dose to the parotids has major implications for the quality life of these patients, as the risk of xerostomia decreases. For every $1 \mathrm{~Gy}$ increase in parotid mean dose, salivary function decreases by 5\% [7]. If at least one parotid gland receives a mean dose of less than $25.8 \mathrm{~Gy}$, the risk of grade 4 xerostomia is significantly lower. As the primary CRT contralateral parotid gland received less than $25.8 \mathrm{~Gy}$ and both adjuvant RT parotid glands received more than $25.8 \mathrm{~Gy}$, we expect the primary CRT cohort to have a significantly lower risk of severe xerostomia, and, therefore, a better quality of life over the long-term.

While most submandibular RTOG 1016 dose targets were achieved for the primary CRT plans, many postoperative RT patients had their submandibular glands removed so dose statistics between the cohorts could not be compared. Though primary CRT delivers radiation to the submandibular glands, the risk of xerostomia due to submandibular irradiation is better than xerostomia from the absence of submandibular glands.

The primary CRT larynx structure had a $48.5 \%$ lower mean dose than adjuvant RT. Caudell et al. [8] found that higher mean doses were significantly associated with severe dysphagia at 12 months post-treatment. Patients began to experience aspiration at a mean dose of $41 \mathrm{~Gy}$ to the larynx. At doses higher than the threshold, the risk of severe dysphagia is significantly correlated with increasing dosage. With the average mean dose of $40.2 \mathrm{~Gy}$ for adjuvant RT and 20.7 Gy for primary CRT, we would expect primary CRT patients to have a lower risk of aspiration. Also, as Caudell et al. [8] only studied primary CRT, postoperative RT patients may experience more severe comorbidities at a mean dose of $41 \mathrm{~Gy}$ as patients irradiated postoperatively suffer lower quality of life and more severe pain with the same dose prescription comparison as our study [4].

Our study's findings show that opting for surgery would not spare advanced oropharyngeal patients of the integral dose and that primary CRT lowers the mean dose to some OARs. Our results also indicated that treating a smaller volume to a higher dose in the primary CRT setting would not increase the risk of developing radiation-related side effects as there is a predicted lower risk of xerostomia and aspiration. In addition to potentially improving quality of life, lower toxicities can decrease appointment and treatment cancellations, which improves outcomes and decreases healthcare costs.

Due to a higher prescribed dose for the primary CRT cohort, we expected higher maximum doses received by many OARs than in the adjuvant RT cohort. The high dose PTV may overlap with some OARs, so maximum doses in those organs are difficult to avoid. The significantly higher maximum dose observed in the primary CRT cohort for the mandible would result in a higher risk of osteoradionecrosis. According to Emami [9], this risk increases above 5\% with a point dose greater than $70 \mathrm{~Gy}$. Thankfully, most of the toxicity in head and neck critical structures are based on mean dose rather than point doses.

There were multiple limitations in our study. The primary CRT cohort was planned on diagnostic CT scans, and therefore the patients were not in a traditional RT position with an immobilizing shell with shoulders depressed and chin extended. This may have resulted in dosimetric differences between the cohorts, with a likely negative effect on the primary CRT 
group's OAR optimization abilities because these scans had compressed anatomy due to the lack of neck extension position in diagnostic scans. Secondly, although treating the postsurgical bed and bilateral neck for locally advanced oropharyngeal patients who have undergone surgery is standard at our center, it may not be so at other centers. There is evidence that postoperative radiotherapy to the ipsilateral neck may be all that's needed for patients with N2a-b disease [10].

\section{Conclusions}

In conclusion, primary CRT offered a lower total integral dose to the neck on average, although this was not statistically significant. Given that primary CRT plans prescribed a higher dose, higher maximum organ doses were expected. However, lower mean organ doses suggested that primary CRT plans spare more larynx and parotid gland than postoperative radiation, which may result in lower overall toxicity to the patient. Randomized clinical trials are necessary to further validate these findings and better inform the management decisions of advanced stage oropharyngeal cancer patients.

\section{Appendices}

\section{Raw Data and Non-2 Gy Fraction Equivalent Doses for Each Patient and Critical Structure}




\section{Cureus}

\begin{tabular}{|c|c|c|c|c|c|c|c|c|}
\hline \multirow{2}{*}{ Patient } & \multicolumn{2}{|c|}{$\begin{array}{l}\text { Max Ipsilateral Parotid } \\
\text { (Gy) }\end{array}$} & \multicolumn{2}{|c|}{$\begin{array}{l}\text { Mean Ipsilateral } \\
\text { Parotid (Gy) }\end{array}$} & \multicolumn{2}{|c|}{$\begin{array}{l}\text { Max Contralateral } \\
\text { Parotid (Gy) }\end{array}$} & \multicolumn{2}{|c|}{$\begin{array}{l}\text { Mean Contralateral } \\
\text { Parotid (Gy) }\end{array}$} \\
\hline & $\mathrm{RT}$ & PO & RT & $\mathrm{PO}$ & RT & PO & $\mathrm{RT}$ & $\mathrm{PO}$ \\
\hline 1 & 75.978 & 63.095 & 38.563 & 54.682 & 54.718 & 57.448 & 25.341 & 37.933 \\
\hline 2 & 74.210 & 64.622 & 36.730 & 38.668 & 53.402 & 65.050 & 25.344 & 37.309 \\
\hline 3 & 75.845 & 67.223 & 36.689 & 45.726 & 53.977 & 64.805 & 25.258 & 39.933 \\
\hline 4 & 73.953 & 62.092 & 30.267 & 37.896 & 61.910 & 60.589 & 24.906 & 30.711 \\
\hline 6 & 76.897 & 68.343 & 25.758 & 40.633 & 70.347 & 64.245 & 25.124 & 37.533 \\
\hline 7 & 73.834 & 59.448 & 39.063 & 37.998 & 56.220 & 54.438 & 25.947 & 19.936 \\
\hline 8 & 75.258 & 64.982 & 51.725 & 50.103 & 54.314 & 60.896 & 25.272 & 26.159 \\
\hline 9 & 69.377 & 64.032 & 25.509 & 34.649 & 51.962 & 60.288 & 24.414 & 26.411 \\
\hline 10 & 75.626 & 66.059 & 40.951 & 32.408 & 54.544 & 55.916 & 25.277 & 24.255 \\
\hline 11 & 79.245 & 65.102 & 34.893 & $36.8 / 1$ & 55.340 & 60.265 & 24.829 & 37.243 \\
\hline 12 & 71.596 & 63.947 & 29.201 & 39.117 & 55.832 & 58.189 & 25.430 & 30.013 \\
\hline 13 & 67.381 & 64.863 & 25.424 & 42.677 & 53.720 & 60.184 & 24.217 & 29.400 \\
\hline 14 & 75.950 & 64.450 & 23.626 & 41.196 & 76.040 & 64.450 & 23.286 & 41.196 \\
\hline 15 & 75.710 & 64.135 & 43.028 & 38.344 & 53.619 & 62.386 & 24.418 & 30.836 \\
\hline 17 & 81.209 & 65.170 & 48.659 & 59.380 & 56.979 & 62.166 & 25.123 & 19.327 \\
\hline 18 & 74.371 & 63.987 & 24.644 & 39.116 & 58.963 & 63.987 & 24.644 & 26.565 \\
\hline 19 & 75.496 & 66.841 & 35.684 & 40.245 & 55.770 & 16.705 & 26.419 & 7.918 \\
\hline 20 & 78.995 & 68.918 & 27.872 & 33.896 & 54.183 & 58.292 & 25.656 & 35.531 \\
\hline
\end{tabular}

TABLE 4: Mean and Maximum Doses to Each Patient's Ipsilateral and Contralateral Parotids for Primary Radiotherapy (RT) and Postoperative Radiotherapy (PO). 


\section{Cureus}

\begin{tabular}{|c|c|c|c|c|c|c|c|c|}
\hline \multirow{2}{*}{ Patient } & \multicolumn{2}{|c|}{ Max Mandible (Gy) } & \multicolumn{2}{|c|}{ Mean Mandible (Gy) } & \multicolumn{2}{|c|}{ Max PC (Gy) } & \multicolumn{2}{|c|}{ Mean PC (Gy) } \\
\hline & RT & PO & RT & $\mathrm{PO}$ & RT & PO & RT & $\mathrm{PO}$ \\
\hline 1 & 68.856 & 65.511 & 31.564 & 44.637 & 75.970 & 64.975 & 55.198 & 60.188 \\
\hline 2 & 73.175 & 64.353 & 37.749 & 46.025 & 76.622 & 65.203 & 55.609 & 60.253 \\
\hline 3 & 75.541 & 64.873 & 38.780 & 39.858 & 74.602 & 65.756 & 55.975 & 60.891 \\
\hline 4 & 74.458 & 63.852 & 42.278 & 37.272 & 74.226 & 63.321 & 64.132 & 59.135 \\
\hline 6 & 77.206 & 63.510 & 46.251 & 46.686 & 76.499 & 64.414 & 66.692 & 61.856 \\
\hline 7 & 75.732 & 63.338 & 42.309 & 43.687 & 74.998 & 63.101 & 60.134 & 59.703 \\
\hline 8 & 75.758 & 65.564 & 44.287 & 35.177 & 76.066 & 64.929 & 60.220 & 61.241 \\
\hline 9 & 76.277 & 63.686 & 47.105 & 40.354 & 72.836 & 63.250 & 54.780 & 52.783 \\
\hline 10 & 75.696 & 65.306 & 39.936 & 47.274 & 75.666 & 64.125 & 54.709 & 53.728 \\
\hline 11 & 77.837 & 64.064 & 40.812 & 49.547 & 74.624 & 65.323 & 56.869 & 57.235 \\
\hline 12 & 63.747 & 63.776 & 33.739 & 42.059 & 75.202 & 64.009 & 56.302 & 59.157 \\
\hline 13 & 60.700 & 64.145 & 32.319 & 45.653 & 76.053 & 63.792 & 55.648 & 60.482 \\
\hline 14 & 76.805 & 63.892 & 45.635 & 47.381 & 74.715 & 64.14 & 60.078 & 61.575 \\
\hline 15 & 75.589 & 64.646 & 40.760 & 45.521 & 75.323 & 63.709 & 59.134 & 60.138 \\
\hline 17 & 79.150 & 65.395 & 44.039 & 46.895 & 76.996 & 63.544 & 56.490 & 55.535 \\
\hline 18 & 76.710 & 64.474 & 45.603 & 41.087 & 80.179 & 60.450 & 57.841 & 48.406 \\
\hline 19 & 76.005 & 65.394 & 37.279 & 31.915 & 73.515 & 64.288 & 55.708 & 37.185 \\
\hline 20 & 75.644 & 65.120 & 35.364 & 36.171 & 75.771 & 63.771 & 58.005 & 52.512 \\
\hline
\end{tabular}

TABLE 5: Mean and Maximum Doses to Each Patient's Mandible and Pharyngeal Constrictors (PC) for Primary Radiotherapy (RT) and Postoperative Radiotherapy (PO) 


\section{Cureus}

\begin{tabular}{|c|c|c|c|c|c|c|c|c|}
\hline \multirow{2}{*}{ Patient } & \multicolumn{2}{|c|}{ Max Larynx (Gy) } & \multicolumn{2}{|c|}{ Mean Larynx (Gy) } & \multicolumn{2}{|c|}{ Max Esophagus (Gy) } & \multicolumn{2}{|c|}{ Mean Esophagus (Gy) } \\
\hline & RT & PO & RT & $\mathrm{PO}$ & RT & PO & RT & PO \\
\hline 1 & 55.211 & 57.797 & 25.828 & 46.85 & 48.947 & 58.157 & 29.360 & 45.744 \\
\hline 2 & 53.781 & 62.123 & 25.256 & 45.202 & 41.628 & 52.123 & 30.268 & 32.025 \\
\hline 3 & 53.460 & 60.269 & 25.091 & 24.870 & 58.375 & 34.771 & 22.414 & 16.311 \\
\hline 4 & 63.436 & 58.689 & 25.963 & 52.500 & 42.979 & 56.451 & 30.257 & 49.662 \\
\hline 6 & 68.064 & 64.414 & 25.584 & 61.856 & 59.767 & 62.881 & 29.832 & 51.394 \\
\hline 7 & 51.546 & 61.068 & 24.969 & 35.542 & 48.679 & 43.772 & 29.852 & 24.903 \\
\hline 8 & 77.158 & 56.392 & 24.675 & 30.797 & 57.147 & 51.000 & 17.937 & 13.933 \\
\hline 9 & 53.470 & 56.194 & 24.474 & 28.057 & 44.746 & 52.477 & 28.725 & 13.721 \\
\hline 10 & 49.455 & 60.581 & 24.688 & 34.448 & 47.729 & 50.196 & 29.819 & 25.512 \\
\hline 11 & 67.345 & 66.255 & 20.277 & 45.123 & 61.083 & 62.817 & 24.290 & 38.646 \\
\hline 12 & 62.682 & 63.936 & 20.934 & 58.526 & 50.893 & 59.882 & 25.541 & 28.115 \\
\hline 13 & 49.980 & 63.268 & 19.560 & 55.714 & 52.023 & 57.241 & 28.577 & 55.714 \\
\hline 14 & 55.298 & 63.130 & 18.920 & 58.253 & 44.076 & 58.117 & 26.263 & 34.192 \\
\hline 15 & 51.699 & 63.292 & 19.633 & 34.621 & 46.290 & 47.428 & 28.781 & 32.260 \\
\hline 17 & 73.034 & 60.214 & 27.990 & 31.747 & 49.850 & 46.782 & 29.616 & 29.198 \\
\hline 18 & 87.904 & 57.702 & 26.171 & 42.294 & 48.628 & 45.403 & 29.392 & 35.469 \\
\hline 19 & 57.484 & 55.129 & 18.715 & 25.358 & 40.465 & 37.482 & 26.139 & 21.093 \\
\hline 20 & 50.654 & 58.446 & 19.079 & 42.728 & 28.671 & 55.548 & 13.233 & 24.896 \\
\hline
\end{tabular}

TABLE 6: Mean and Maximum Doses to Each Patient's Larynx and Esophagus for Primary Radiotherapy (RT) and Postoperative Radiotherapy (PO) 


\begin{tabular}{|c|c|c|c|c|c|c|c|c|}
\hline \multirow{2}{*}{ Patient } & \multicolumn{2}{|c|}{ Max Cord (Gy) } & \multicolumn{2}{|c|}{ Mean Cord (Gy) } & \multicolumn{2}{|l|}{ ID (Gy/L) } & \multicolumn{2}{|c|}{ PTV (cc) } \\
\hline & RT & $\mathrm{PO}$ & RT & $\mathrm{PO}$ & RT & $\mathrm{PO}$ & RT & PO \\
\hline 1 & 46.862 & 43.608 & 37.008 & 27.592 & 96.20714 & 108.7386 & 61.7 & 442.3 \\
\hline 2 & 44.382 & 42.434 & 31.831 & 22.836 & 114.82917 & 133.9186 & 166.2 & 448.9 \\
\hline 3 & 44.891 & 43.280 & 34.376 & 20.895 & 115.65425 & 120.4623 & 174.7 & 390.5 \\
\hline 4 & 44.243 & 42.610 & 29.730 & 21.671 & 129.98775 & 135.6595 & 117.0 & 121.2 \\
\hline 6 & 46.612 & 42.823 & 37.577 & 26.060 & 143.41090 & 150.5132 & 315.5 & 981.7 \\
\hline 7 & 43.399 & 40.033 & 34.694 & 18.327 & 120.03611 & 92.2390 & 146.0 & 272.7 \\
\hline 8 & 46.314 & 44.487 & 29.391 & 27.487 & 129.75098 & 129.1895 & 267.9 & 496.2 \\
\hline 9 & 44.649 & 39.295 & 36.507 & 21.422 & 137.63596 & 135.1606 & 157 & 297.8 \\
\hline 10 & 43.798 & 43.371 & 33.189 & 21.675 & 97.36787 & 101.2248 & 139.4 & 443.8 \\
\hline 11 & 45.685 & 43.002 & 31.444 & 26.922 & 77.82079 & 85.48116 & 110.3 & 774.6 \\
\hline 12 & 46.341 & 42.588 & 34.935 & 26.45 & 117.54916 & 123.2601 & 171.2 & 378.3 \\
\hline 13 & 46.765 & 44.536 & 35.079 & 31.503 & 111.76074 & 127.5994 & 112.9 & 332 \\
\hline 14 & 43.081 & 42.441 & 34.711 & 21.151 & 177.38555 & 157.9239 & 484.2 & 836.3 \\
\hline 15 & 43.744 & 44.211 & 36.784 & 22.196 & 161.03830 & 174.4947 & 276.9 & 667.1 \\
\hline 17 & 47.43 & 42.632 & 35.924 & 22.762 & 137.34864 & 166.7237 & 302.2 & 462.8 \\
\hline 18 & 45.645 & 43.011 & 37.099 & 20.235 & 192.49248 & 170.0389 & 115.3 & 400.7 \\
\hline 19 & 47.234 & 44.071 & 35.906 & 15.803 & 111.88793 & 77.28451 & 72.1 & 212.8 \\
\hline 20 & 45.21 & 43.58 & 32.594 & 26.04 & 161.80963 & 140.346 & 181.6 & 433.6 \\
\hline
\end{tabular}

\section{TABLE 7: Mean and Maximum Doses to Each Patient's Spinal Cord, Integral Dose} (ID), and PTV size for Primary Radiotherapy (RT) and Postoperative Radiotherapy (PO)

\section{Additional Information \\ Disclosures}

Human subjects: Consent was obtained by all participants in this study. Animal subjects: All authors have confirmed that this study did not involve animal subjects or tissue. Conflicts of interest: In compliance with the ICMJE uniform disclosure form, all authors declare the following: Payment/services info: All authors have declared that no financial support was received from any organization for the submitted work. Financial relationships: All authors have declared that they have no financial relationships at present or within the previous three 
years with any organizations that might have an interest in the submitted work. Other relationships: All authors have declared that there are no other relationships or activities that could appear to have influenced the submitted work.

\section{Acknowledgements}

We would like to thank Naomi Parker, Brian Chwyl, Kim Rans, Andree Desrochers, and Laura Grose for their help with this project.

\section{References}

1. Odell MJ, Walz BJ, Reimers H, et al.: Carcinoma of the oropharynx. Head and Neck Cancer. Thieme Medical Publishers, Inc (ed): New York, NY, 2008; 2008. 24-44.

2. Chen LA, Anker CJ, Hunt JP, et al.: Clinical outcomes associated with evolving treatment modalities and radiation techniques for base-of-tongue carcinoma: thirty years of institutional experience. Cancer Med. 2015, 4:651-660. 10.1002/cam4.364

3. Parsons JT, Mendenhall WM, Stringer SP, et al.: Squamous cell carcinoma of the oropharynx: surgery, radiation therapy, or both. Cancer. 2002, 94:2967-2980. 10.1002/cncr.10567

4. Boscolo-Rizzo P, Stellin M, Fuson R, et al.: Long-term quality of life after treatment for locally advanced oropharyngeal carcinoma: surgery and postoperative radiotherapy versus concurrent chemoradiation. Oral Oncol. 2009, 45:953-957.

10.1016/j.oraloncology.2009.06.005

5. O’Connell D, Seikaly H, Murphy R, et al.: Primary surgery versus chemoradiotherapy for advanced oropharyngeal cancers: a longitudinal population study. Otolaryngol Head Neck Surg. 2013, 42:31. 10.1186/1916-0216-42-31

6. Tillman GF, Pawlicki T, Koong AC, et al.: Preoperative versus postoperative radiotherapy for locally advanced gastroesophageal junction and proximal gastric cancers: a comparison of normal tissue radiation doses. Dis Esophagus. 2008, 21:437-444.

7. Blanco AI, Chao KC, El Naqa I, et al.: Dose-volume modeling of salivary function in patients with head-and-neck cancer receiving radiotherapy. Int J Radiat Oncol Biol Phys. 2005, 62:1055-1069. 10.1016/j.ijrobp.2004.12.076

8. Caudell JJ, Schaner PE, Meredith RF, et al.: Factors associated with long-term dysphagia after definitive radiotherapy for locally advanced head-and-neck cancer. Int J Radiat Oncol Biol Phys. 2009, 73:410-415. 10.1016/j.ijrobp.2008.04.048

9. Emami B, Lyman J, Brown A, et al.: Tolerance of normal tissue to therapeutic ratio . Int J Radiat Oncol Biol Phys. 1991, 21:109-122. 10.1016/0360-3016(91)90171-Y

10. Vergeer MR, Doornaert PA, Jonkman A, et al.: Ipsilateral irradiation for oral and oropharyngeal carcinoma treated with primary surgery and postoperative radiotherapy. Int J Radiat Oncol Biol Phys. 2010, 78:682-8. 10.1016/j.ijrobp.2009.08.042 